\title{
A Study of Perturbations in Structure and Elastic Modulus of Bone Microconstituents Using Bimodal Amplitude Modulated-Frequency Modulated Atomic Force Microscopy
}

\author{
Yao Sun, ${ }^{\dagger}$ Lien Hong Vu, ${ }^{\ddagger}$ Nicholas Chew, ${ }^{\ddagger}$ Zudin Puthucheary, ${ }^{\ddagger}{ }^{\S}$ Matthew E. Cove, \\ and Kaiyang Zeng*, ${ }^{\dagger}$ \\ ${ }^{\dagger}$ Department of Mechanical Engineering, National University of Singapore, 9 Engineering Drive 1, 117576, Singapore \\ ${ }^{\ddagger}$ Department of Medicine, Yong Loo Lin School of Medicine, National University of Singapore, 1E Kent Ridge Road, NUHS Tower \\ Block Level 10, Singapore 119228 \\ ${ }^{\S}$ Division of Critical Care, Institute of Sports and Exercise Health, University College London Hospitals, U.K., and Centre for \\ Human Health and Performance, University College London, London WC1E 6BT, United Kingdom
}

Supporting Information

ABSTRACT: Sepsis-related bone diseases are rarely reported although many ICU patients are diagnosed with bone damage after prolonged immobility. In this work, cortical bone of femurs from Sprague-Dawley rats under mild sepsis condition are investigated by using Scanning Probe Microscopy (SPM) to study the influence of sepsis on the changes of structure, chemistry, and elastic modulus of bone microconstituents, i.e., collagen fibers and mineral. The results show that there are significant changes on elastic modulus, shape, and chemical composition of collagen fibers $24 \mathrm{~h}$ after the sepsis insult, but all of the changes are recovered to almost normal $96 \mathrm{~h}$ after the insult. These phenomena are found to

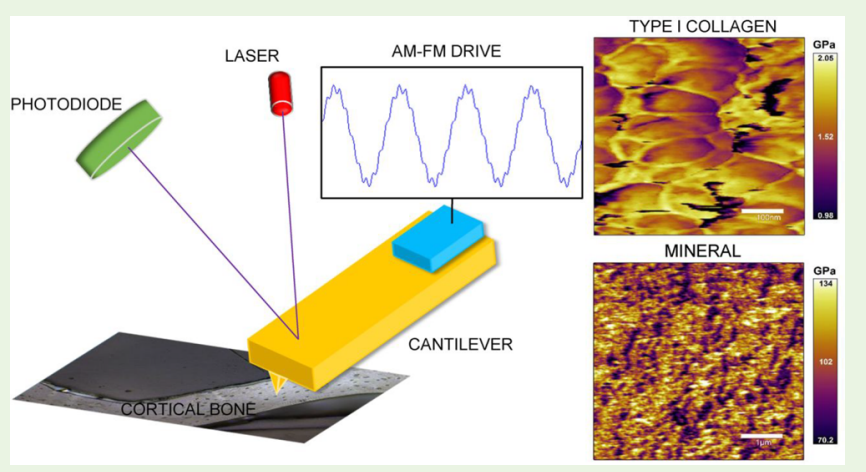
be associated with demineralization of the collagen fiber. For the mineral constituents in bone, the elastic modulus decreases significantly $96 \mathrm{~h}$ after the insult, showing slower responses compared with those in the collagen fibers. Particle analysis reveals that the size of the mineral particles decreases continuously and significantly with the time after the sepsis insulting. This work reveals the responding processes of bone microconstituents to sepsis in rat mode and, hence, can provide an insight into the pathogenesis of sepsis related human bone damage.

KEYWORDS: elastic modulus, bone, collagen, mineral, bimodal atomic force microscopy

\section{INTRODUCTION}

Bone is a natural nanocomposite material forming the skeleton of the body. The microconstituents of bone are tissues and proteins (organic phase components), minerals (inorganic phase components), and water. Typically, the organic phase consists of biomacromolecules (type I collagen and noncollagenous proteins) while the inorganic phase mainly consists of hydroxyapatite (HA) crystals. $^{1}$ As a result of the complex hierarchical structures consisting of both biomacromolecules and minerals with certain porosity, ${ }^{2}$ bone is stiff and tough in general. $^{3-5}$ Bone constituent analysis is normally divided into four size scales: nanoscale (measured in nanometers), submicroscale (one to tens of microns), microscale (tens to hundreds of microns) and mesoscale (hundreds of microns to millimeters or more). ${ }^{6}$ Nanoscale analysis demonstrates that Type I collagen occupies $90 \%$ of the organic phase in bone. Type I collagen molecules and HA crystals are arranged in a staggered pattern to form mineralized collagen fibers that are approximately $100-200 \mathrm{~nm}$ in diameter. ${ }^{8}$ In bone structures, the mineralized collagen fibers are embedded in the mineral matrix.

Sepsis is a clinical condition that occurs when the body's inflammatory response to infection results in multiorgan damage and/or failure. ${ }^{9}$ It was reported that sepsis could cause bone density loss and increase the risk of bone fracture. ${ }^{10-12}$ At present, BMD (bone mineral density) is the gold-standard test for bone loss. However, one limitation is its inability to examine changes of the nanoscale structure as well as the mechanical strength of the bone, both are vital components of the bone health. From the engineering point of view, techniques frequently applied for assessing the structure of bones include optical/electronic microcopy, X-ray diffraction (XRD), ${ }^{13}$ and Raman spectroscopy, ${ }^{13,14}$ but these techniques have significant disadvantages in terms of radiation damage and relative low

Received: September 11, 2018

Accepted: December 14, 2018

Published: December 14, 2018 
resolution (submicroscale). It has been reported that ultrasound waves can be used to assess elastic properties of cancellous bone, ${ }^{15}$ but the accuracy of this technique is unclear. Nanoindentation, the most widely used experimental method, can be used to assess the bone's elastic modulus and hardness at submicroscale level, ${ }^{16-18}$ but with difficulties in separating the mechanical properties of collagen and mineral. Further, it can lead to local plastic yielding and permanent deformation of the sample. ${ }^{19}$ Atomic force microscopy (AFM) can be used to characterize the mechanical properties with a nanometer spatial resolution but with minimal applied force, usually in the order of nano-Newtons $(\mathrm{nN}) .^{20}$ In addition to the extensively used single point AFM-based nanoindentation method, ${ }^{21-24}$ Wenger et al. used the time-consuming force volume mapping to visualize the stiffness of single type I collagen fibers. ${ }^{25}$ Campbell et al. successfully adopted contact resonance force microscopy (CRFM) technique to map the nanostructure and transformed elasticity image from contact resonance frequency image of the osteochondral interface. ${ }^{26}$ In the past few years, the multifrequency AFM techniques ${ }^{27-37}$ (including bimodal AFM), where two or more eigenmodes of the probe cantilever are simultaneously excited during the AFM scan, have emerged and attracted many attention. Through exciting higher harmonic of the AFM cantilever, bimodal AFM provides fast imaging of morphology as well as elastic property of materials at the same tine. ${ }^{38}$ According to the selection of either amplitude modulation (AM) or frequency modulation (FM), bimodal AFM can be divided into AM-AM mode, AM-FM mode, and FM-FM mode. ${ }^{39}$ For example, the newly developed AM-FM approach realizes high-resolution scanning images of materials via amplitude tapping mode (topography by AM) and highsensitivity frequency modulation mode (elasticity by $\mathrm{FM}) .{ }^{39} \mathrm{Li}$ et al. recently reported that collagen fibers from an osteogenesis imperfecta mouse (oim) model exhibit significantly lower mean stiffness and smaller deviation than those in the wild-type mouse using the advanced bimodal AM-FM technique. ${ }^{40} \mathrm{~A}$ recent study has investigated the effects of sepsis to the mechanical properties of bone by using multiple techniques including AMFM. ${ }^{11}$ It was observed that the collagen and mineral responses to the sepsis insult in the different time scales. ${ }^{11}$ In this study, we will therefore further quantify the change of nanoscale structure and elastic modulus of both collagen fibers and minerals of the femur cortical bones from both sepsis and control wild-type rats using the AM-FM technique. By using the AM-FM technique, topography, and stiffness images can be obtained simultaneously. It is reported that the stiffness obtained in dynamic AFM measurements depends on both the elastic modulus of the sample and the geometry of the AFM tip. ${ }^{41,42}$ In addition, the geometry of AFM tip may change during the contact mode scanning, which can affect measurement accuracy of the stiffness. ${ }^{42}$ To improve the measurement accuracy, we adopt appropriate reference material and Hertz contact model ${ }^{43}$ to derive the elastic modulus map from the AM-FM stiffness map without determination of tip and tip-sample contact radii (Supporting Information (SI)). After each scan of bone samples, the reference material is immediately scanned with the same tip to ensure that the tip geometry remains unchanged at utmost. It should be noted that the elastic modulus of the reference material should be similar to that of the test sample so that the contact radii of tip-sample and tip-reference material systems can be treated to be equal. ${ }^{39}$ To the best of our knowledge, this work is the first and complete nanoscale visualization of elastic modulus and structure features of both collage fibers and minerals of the bone samples from the sepsis model.

\section{MATERIALS AND METHODS}

2.1. Animal Preparation. Twelve-week old male SD rats (300$350 \mathrm{~g}$ ) were used in the mild sepsis mode, which were housed and given access to food and drink ad libitum for at least 3 days to allow for acclimatization. Then, the rats were randomly divided into one sham and two Cecal Ligation Puncture (CLP) groups. After sepsis insult, rats were housed individually. The two CLP groups were euthanized after 24 and $96 \mathrm{~h}$ of the insult, respectively. (The sepsis rat-mode was developed under the guidelines of the National University of Singapore (NUS) Institutional Animal Care and Use Committee (Permit Number: IACUC R14-1434) at School of Medicine, NUS under the support of the Eng-Med collaborative project.)

2.2. Cecal Ligation and Puncture. Rats were anesthetized by inhalation with 2 to $5 \%$ Isoflurane (Baxter Healthcare Corporation) in $100 \%$ oxygen using anesthesia equipment. A $2 \mathrm{~cm}$ long midline incision was performed through the skin and then through the linea alba under sufficient anesthesia. The cecum was identified and lifted out of the peritoneal cavity. In the sham group, the cecum was replaced without ligation or puncture. While in the CLP groups, the cecum was ligated tightly in a manner that conserved bowel continuity and punctured in a single pass through the anterior and posterior walls with an $18 \mathrm{G}$ needle (Terumo). Then the laparotomy was closed with suture (B. Braun). At the end of the operation, the animals were given fluid resuscitation and analgesia and returned to individual cages. Femurs were collected after 24 and $96 \mathrm{~h}$ of the insult, respectively. Hence, the three types of bone samples are collected and named as sham, $24 h C L P$, and $96 h C L P$, respectively.

2.3. Sample Preparation. In preparation of the samples for the AFM measurements, the femur bones of the sham and two CLP groups were rinsed thoroughly and adherent soft tissue was scraped away carefully. Femurs were sectioned by a slicer (Struers, Fuse 2, 5 AT, Denmark) and the middle part was kept for measurements with thickness of approximately $10 \mathrm{~mm}$. Subsequently the bones were left to dry in air for an hour and then embedded in epoxy resin (AMET, India). In order to increase its viscosity and avoid the infiltration of resin into the pores, the epoxy resin and hardener were mixed and allowed to cool down for 10 min prior to embedding the bone samples. The bones with resins were left to polymerize at room temperature. After polymerization of the epoxy resin, the bone samples were sequentially polished using increasing grades of carbide papers (P800-P4000) and further polished with 0.3 and $0.05 \mu \mathrm{m}$ alumina powder. Samples were then sonicated in acetone to remove the residual epoxy resin surrounding the samples. Finally, the samples were air-blown dried.

2.4. Principle of Bimodal Atomic Force Microscopy. By simultaneously exciting the second or higher eigen-frequency, bimodal atomic force microscopy can achieve fast imaging of sample topography, elastic, and composition information on materials. ${ }^{44}$ Simultaneous actuating of two or more eigenfrequencies can improve the coupling of different modes. ${ }^{45}$ Combination of the feedbacks of the first resonance amplitude (AM) and the second resonance frequency shift (FM) are known as AM-FM technique. ${ }^{46}$ The principle of AMFM mode of bimodal AFM and the setup are shown in Figure S1 (SI). In this work, the AM-FM technique is based on the commercial AFM system (MFP-3D, Asylum Research, Oxford Instruments, CA, U.S.A.). The tip-sample interaction was set in the repulsive regime (phase $<90^{\circ}$ ) in order to achieve a certain indentation depth (usually a few nanometers) into the sample surface. The drive amplitudes for the first and second eigenmode were $\sim 105$ and $\sim 0.5 \mathrm{~nm}$, respectively. The much smaller drive amplitude for second eigenmode was chosen to avoid the perturbations to the first eigenmode cantilever oscillation. ${ }^{40}$

\section{RESULTS AND DISCUSSION}

3.1. AM-FM Characterization of Collagen Fiber. The commercial AFM probe (AC200TS, Asylum Research, Oxford Instruments, CA, U.S.A.) with calibrated first eigenmode spring 
(a)

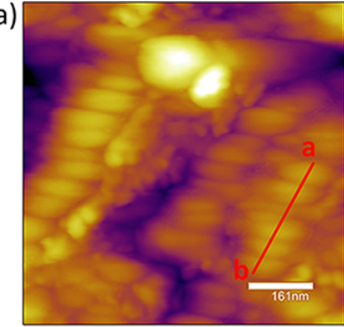

(d)

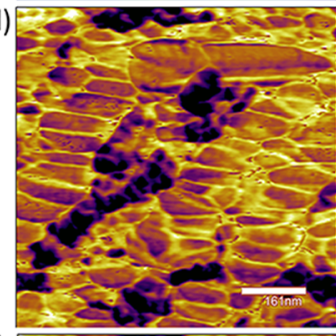

(g)

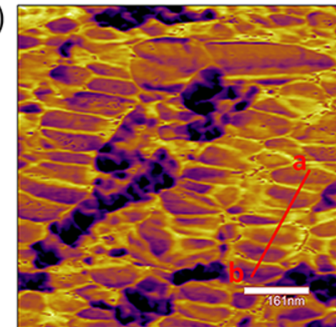

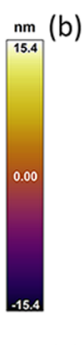

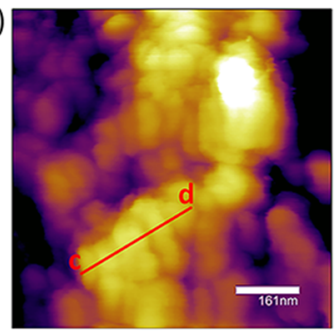

$\mathrm{N} / \mathrm{m}(\mathrm{e})$

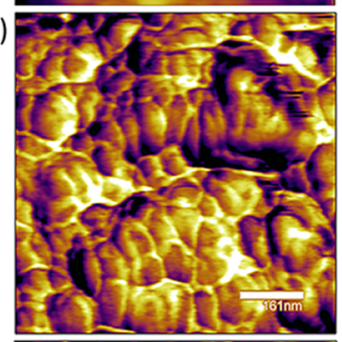

GPa $(h)$

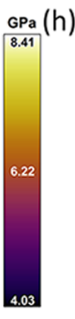

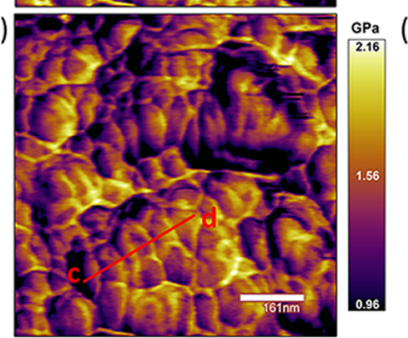
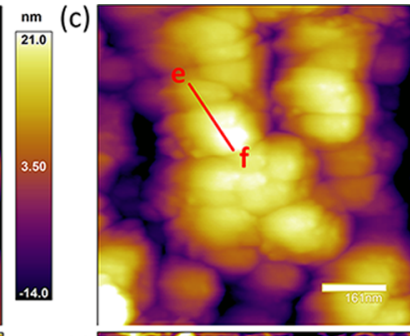

$\mathrm{mN/m}(\mathrm{f})$
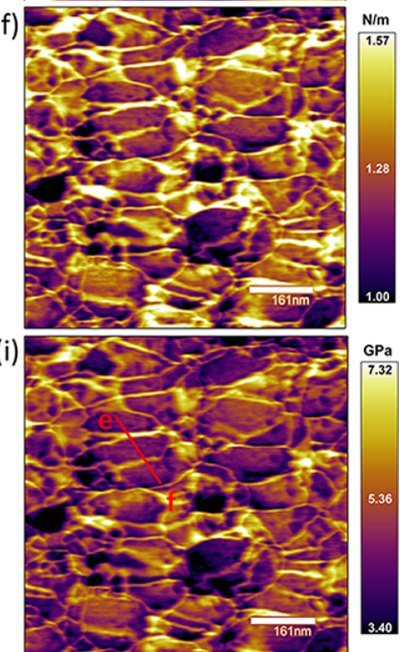

Figure 1. AM-FM images $(800 \mathrm{~nm} \times 800 \mathrm{~nm})$ of collagen fibers in the sham (first column), $24 \mathrm{~h} C L P$ (second column), and $96 \mathrm{~h} C L P$ (third column) cortical bones, respectively. $(\mathrm{a}-\mathrm{c})$ Topography, $(\mathrm{d}-\mathrm{f})$ stiffness, and $(\mathrm{g}-\mathrm{i})$ calculated elastic modulus images, respectively.

Table 1. Summary of the Quantified Data from AFM Measurements

\begin{tabular}{lll} 
& \multicolumn{1}{c}{ sham } & \multicolumn{2}{c}{ CL $h$ CLP } \\
collagen roughness (polished samples) & $3.99 \mathrm{~nm}$ & $9.11 \mathrm{~nm}$ \\
collagen contact stiffness (from AM-FM image) & $1.17-1.79 \mathrm{~N} / \mathrm{m}$ & $396-696 \mathrm{mN} / \mathrm{m}$ \\
collagen modulus (calculated) & $4.03-8.41 \mathrm{GPa}$ & $0.96-2.16 \mathrm{GPa}$ \\
mineral roughness (polished sample) & $15.95 \mathrm{~nm}$ & $20.61 \mathrm{~nm}$ \\
mineral contact stiffness (from AM-FM image) & $0.85-1.59 \mathrm{~N} / \mathrm{m}$ & $0.77-1.47 \mathrm{~N} / \mathrm{m}$ \\
mineral modulus (calculated) & $68.1-144 \mathrm{GPa}$ & $70.2-134 \mathrm{GPa}$ \\
calcium contents (EDS) & $2.8 \%$ & $4.96 \%$ \\
particle height (from AFM image) & $18.38 \pm 2.13 \mathrm{~nm}$ & $15.74 \pm 0.85 \mathrm{~nm}$ \\
particle percentage (from AFM image) & $58.7 \%$ & $60.6 \%$ \\
average particle area (from AFM image) & $0.36 \mu \mathrm{m}^{2}$ & $0.41 \%$ \\
\end{tabular}

constant of $8.98 \mathrm{~N} / \mathrm{m}$ by the thermal noise method is used to characterize the collagen fibers. More specific probe parameters are summarized in the Table S1 (SI). A polyethylene terephthalate (PET) wafer (with elastic modulus of $3.599 \mathrm{GPa}$ (determined from nanoindentation experiments) is chosen as the reference material in order to determine the elastic modulus of the collagen fibers. It is worth noticing that the nondimensional photodiode sensitivity $(m)$ of the AFM, which is defined as the inverse of the slope (volts/volts) of the deflection versus $\mathrm{Z}$-piezo displacement curve in the repulsive regime of the interaction, can cause the experimental uncertainty in determination of the elastic modulus in the study (SI). ${ }^{47}$ Hence, we perform the uncertainty analyses on the results of the collagen fibers of the sham, $24 h C L P$, and $96 h C L P$ bone samples based on 40 force-Z-piezo distance (F-Z) curves with $95 \%$ confidence interval (CI), respectively, the results are shown in Table S2 (SI). All the AM-FM images are composed of $256 \times$ 256 scanning points. The topography, stiffness, as well as the calculated elastic modulus images of collagen fibers in cortical bone of the sham and two CLP bone samples are shown in Figure
1 and the related data can be found in Table 1. In addition, Figure S3 (SI) offers an extra set of AM-FM results of collagen fibers from different collagens in the three groups of bone samples.

As shown in the topography images (Figure $1 \mathrm{a}-\mathrm{c}$ ), the measured height differences of the collagen fibers on the polished bone surfaces are about $30.8,35.0$, and $31.0 \mathrm{~nm}$ for the sham, $24 h C L P$, and $96 h C L P$ bone samples, respectively. The roughness $(\mathrm{Ra})$ values are $3.99,9.11$, and $7.39 \mathrm{~nm}$ accordingly (as shown in Table 1) by using commercial software from the AFM system (Igor Pro 6.3.7, WaveMetrics, OR, U.S.A.). In the additional topography images showed in Figure S3 (SI), the measured height differences are 24.0, 15.0 and $35.8 \mathrm{~nm}$ for the sham, $24 h$ CLP, and 96 CLP bone samples with Ra of 4.54, 2.76, and $8.64 \mathrm{~nm}$, accordingly. The measured height may be due to both morphological height and the changes in chemistry of the samples. By conducting 30 force measurements (Figure $\mathrm{S} 4, \mathrm{SI}$ ) in the noninvasive attractive regime on the collagen fibers from the sham, $24 h C L P$, and $96 h C L P$ bone samples, we have obtained their Hamaker constants $(H)$ and the minimum 

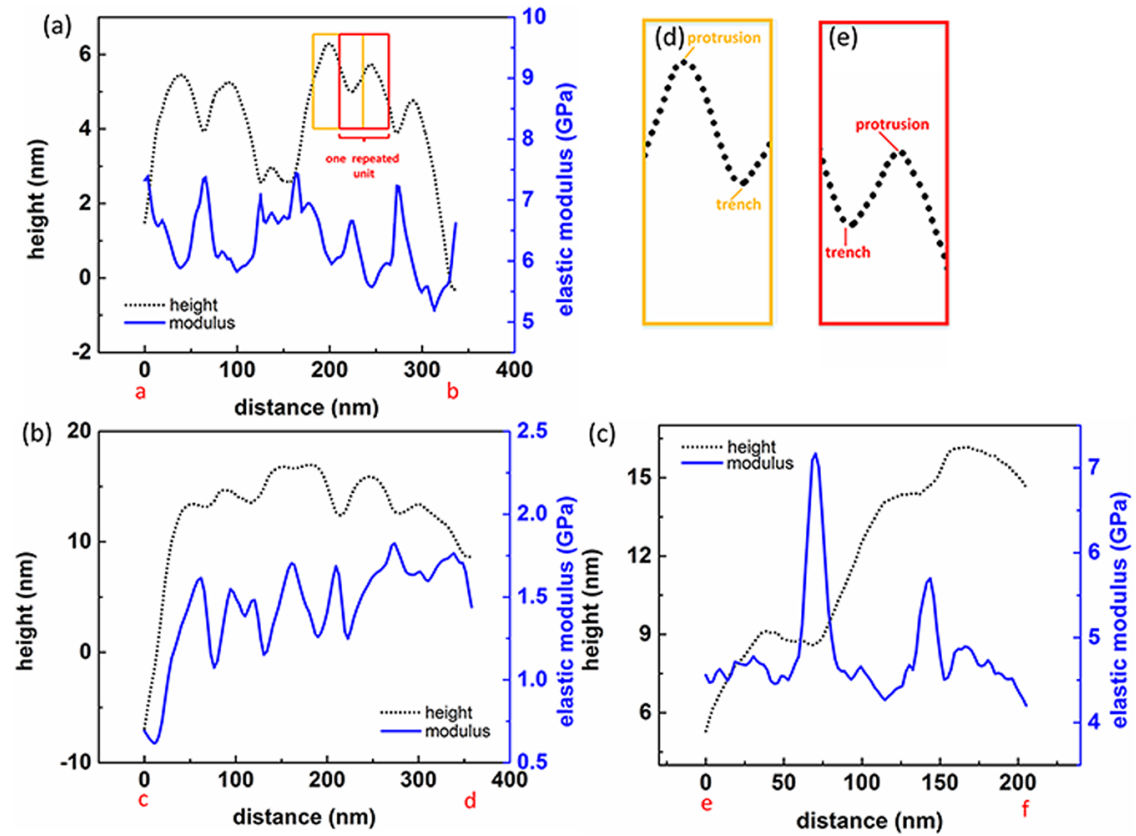

Figure 2. Corresponding height and elastic modulus line profiles along the lines in Figure 1 (a) along the line a-b in Figure 1a, (b) along the line $c-d$ in Figure $1 \mathrm{~b},(\mathrm{c})$ along the line $\mathrm{e}-\mathrm{f}$ in Figure $1 \mathrm{c}$, respectively. (d,e) The protrusion and trench zones of the collagen fiber along the line profile in (a).

distances of approach per cycle $\left(d_{\text {min }}\right)$ in which it can be interpreted as the chemical change and height loss due to the chemistry heterogeneity. ${ }^{48-51}$ The detailed calculation procedures can be found in the corresponding section of the Supporting Information (SI). The $H$ constants are 0.037, 0.173 , and 0.025 atto $J$, and the $d_{\min }$ values are $0.15,0.41$, and $0.12 \mathrm{~nm}$ for the collagen fibers for the sham, $24 \mathrm{hCLP}$, and $96 \mathrm{~h}$ CLP bone samples, respectively. The $d_{\min }$ values are about $0.49 \%, 1.17 \%$, and $0.39 \%$ of the measured height of collagen fibers in AM-FM topography images for sham, 24h CLP, and $96 h$ CLP bone samples. The larger $H$ contant $(0.173$ atto $J)$ and $d_{\text {min }}$ value $(0.41 \mathrm{~nm})$ demonstrate that the chemical change and height loss due to chemistry heterogeneity are more significant in collagen fibers from the $24 h C L P$ bone sample than those from the sham and $96 h C L P$ bone samples. In addition, it is noticeable that all the collagen fibers from the sham, $24 \mathrm{~h} \mathrm{CLP}$, and $96 h C L P$ bone samples display the characteristic $\mathrm{D}$-periodic structures. $^{52,53}$ Interestingly, the collagen fibers from the $24 \mathrm{~h}$ CLP bone sample show curved shape compared to the straight shape of the collagen fibers from the sham and $96 h$ CLP bone samples [Figure 1a-c, and Figure S3a-c (SI)]. The curved shape of the collagen fibers observed from the $24 h$ CLP bone sample most likely indicates that the shape changes in the collagen fibers of bone due to the sepsis insult. It is expected that such shape changes is due to the reduction of mechanical strength of the collagen fibers. This is further revealed from the stiffness images (Figure $1 \mathrm{~d}-\mathrm{f}$ ) showing that the collagen fibers from $24 h C L P$ bone samples have much lower contact stiffness $(396-696 \mathrm{mN} / \mathrm{m})$ than those from the $\operatorname{sham}(1.17-1.79 \mathrm{~N} / \mathrm{m})$ and $96 h$ CLP bone samples $(1.0-1.57 \mathrm{~N} / \mathrm{m})$ (Table 1$)$. The additional stiffness images (Figure $3 \mathrm{~d}-\mathrm{f}, \mathrm{SI}$ ) also show the collagen fiber from the $24 h C L P$ bone has much lower contact stiffness $(306-536 \mathrm{mN} / \mathrm{m})$ than those from the sham $(1.22-$ $1.85 \mathrm{~N} / \mathrm{m})$ and $96 h C L P$ bones $(0.76-1.63 \mathrm{~N} / \mathrm{m})$. Figure $1 \mathrm{~g}-\mathrm{i}$ are the images of calculated elastic modulus. It can be observed that the elastic modulus of collagen fibers shows a trend of decreasing from 4.03 to $8.41 \mathrm{GPa}$ (the sham bone sample) to
0.96-2.16 GPa (24h CLP bone sample) and then increasing to 3.40-7.32 GPa (96 $h$ CLP bone sample) (Table 1). In Figure $\mathrm{S} 3 \mathrm{~g}-\mathrm{i}(\mathrm{SI})$, the elastic modulus decreases from 4.8 to $8.2 \mathrm{GPa}$ (the sham bone) to $0.98-2.05 \mathrm{GPa}(24 h \mathrm{CLP}$ bone) and then increases to $4.35-7.65 \mathrm{GPa}$ (96h CLP bone). It is found that the calculated elastic moduli of collagen fibers from the sham, $24 \mathrm{~h}$ $C L P$, and $96 h C L P$ bone samples are close to the values reported in the literatures. ${ }^{25,54}$ The uncertainty analyses (Table S2, SI) reveal that the uncertainty values are $1.45,0.64$, and $2.10 \mathrm{GPa}$ whereas the uncertainty propagation values are $2.89,1.28$, and 4.20 GPa due to the changes of $m$ values for collagen fibers from the sham, $24 h C L P$, and $96 h C L P$ bone samples, respectively.

For statistical purposes, we plot the elastic moduli of collagen fibers in 10 different AM-FM images (each contains 1-3 collagen fibers) from 10 different femurs for the sham, $24 \mathrm{~h}$ CLP, and $96 \mathrm{~h} \mathrm{CLP}$ bones respectively in Figure S5 (SI). In total, 2030 collagen fibers are randomly selected for statistical analysis. It can be observed that the collagen fibers from $24 h$ CLP bones (Figure S5b, SI) have lower elastic moduli than those from the sham bones (Figure S5a, SI) and the $96 h$ CLP bones (Figure S5c, SI).

3.2. Nanoscale Structure Analysis of Collagen Fiber. We further plot the corresponding measured height and elastic modulus line profiles (Figure 2) along the lines $a-b, c-d$, and $\mathrm{e}-\mathrm{f}$ in Figure $\mathrm{la}-\mathrm{c}$. For the collagen fibers from the sham and 24 $h$ CLP bones, the measured height protrusions have local minimums of elastic modulus while the measured trenches height have the local maximums of elastic modulus, which demonstrates the trench zone (expected to be gap zone) is generally stiffer than that in the protrusion zone (overlap zone), and this observation agrees with what reported in the literature. ${ }^{40}$ However, the correspondence of measured height and elastic modulus can be hardly seen in the collagen fiber in 96 $h$ CLP bone. This finding suggests that the nanostructure of collagen fiber is still affected even $96 \mathrm{~h}$ after the sepsis insult. On the basis of those AM-FM experiments, it is expected that the mechanical property of collagen fiber decreases $24 \mathrm{~h}$ after sepsis 
(a)

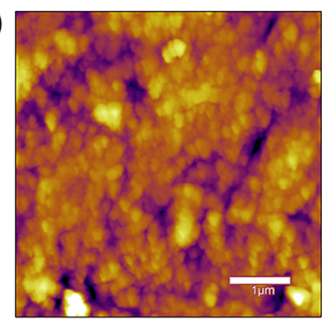

(d)

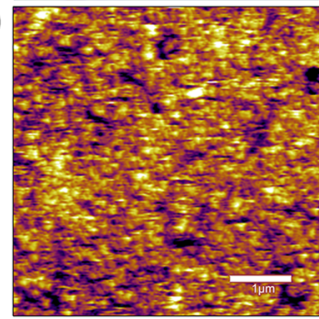

(g)

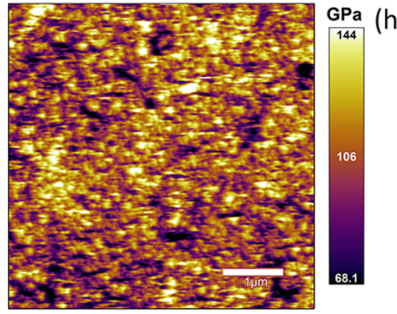

${ }^{\mathrm{nm}}(\mathrm{b})$
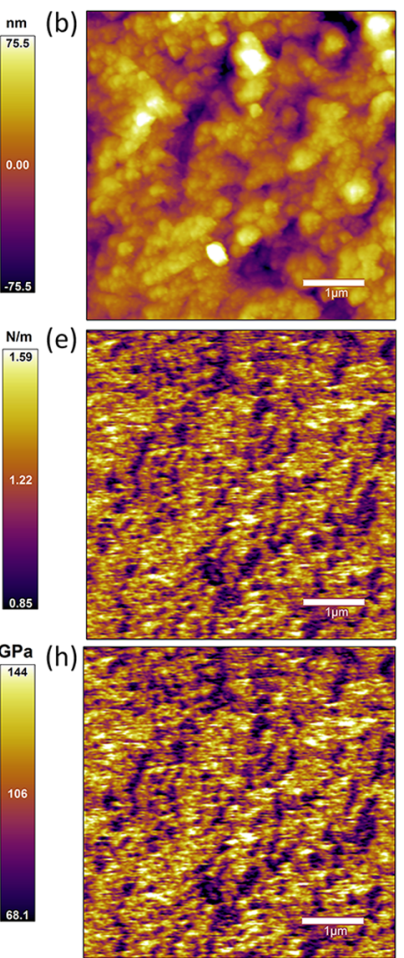
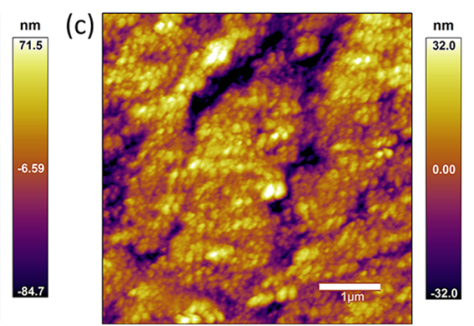

$\mathrm{N} / \mathrm{m}$ (f)

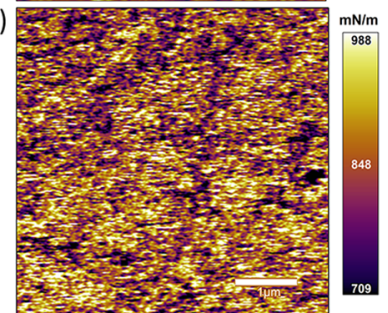

(i)

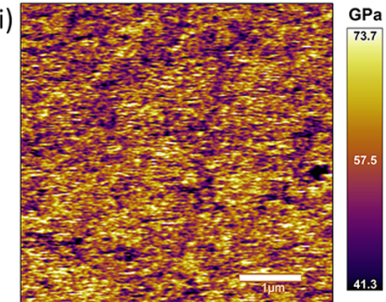

Figure 3. AM-FM images $(5 \mu \mathrm{m} \times 5 \mu \mathrm{m})$ of mineral in the sham (first column), $24 h C L P$ (second column), and $96 h$ CLP (third column) cortical bones, respectively. $(\mathrm{a}-\mathrm{c})$ Topography, $(\mathrm{d}-\mathrm{f})$ stiffness, and $(\mathrm{g}-\mathrm{i})$ calculated elastic modulus images, respectively.
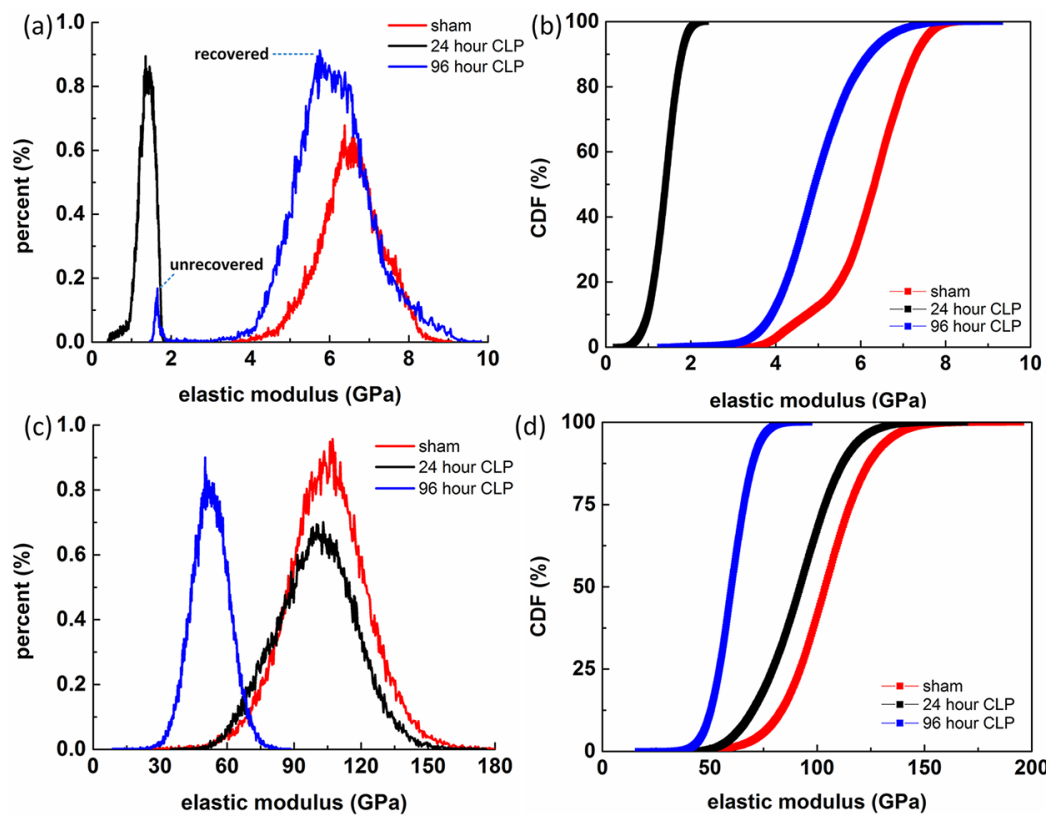

Figure 4. (a) Histograms of the elastic modulus of the collagen fibers from the calculated elastic modulus images in Figure 1g-i. (b) The average CDF charts $(p<0.01)$ of 20-30 collagen fibers from 10 different femur bone samples. (c) Histograms of the elastic modulus of the mineral from the images in Figure $3 \mathrm{~g}-\mathrm{i}$. (d) The average CDF charts $(p<0.01)$ from 10 mineral images obtained from 10 different femur bone samples, respectively.

insult whereas the nanostructure continuously changes even 96 $\mathrm{h}$ after the sepsis insult.

3.3. AM-FM Characterization of Bone Mineral. The commercial AFM probe (AC160TS, Asylum Research, Oxford Instruments, CA, U.S.A.) with calibrated first eigenmode spring constant of $38.5 \mathrm{~N} / \mathrm{m}$ by the thermal noise method is adopted to characterize the bone mineral. More specific parameters of this probe can be found in Table S3 (SI). A wafer of pure tin (with elastic modulus value of $50 \mathrm{GPa}$ measured by nanoindentation experiments) is used as the reference material to determine the elastic modulus of the mineral. The uncertainty analyses on the elastic modulus of the mineral from the sham, $24 \mathrm{~h} \mathrm{CLP}$, and $96 \mathrm{~h}$ CLP bone samples based on 40 F-Z curves with 95\% CI are shown in Table S2 (SI). Figure 3 shows the AM-FM images of the bone mineral. 

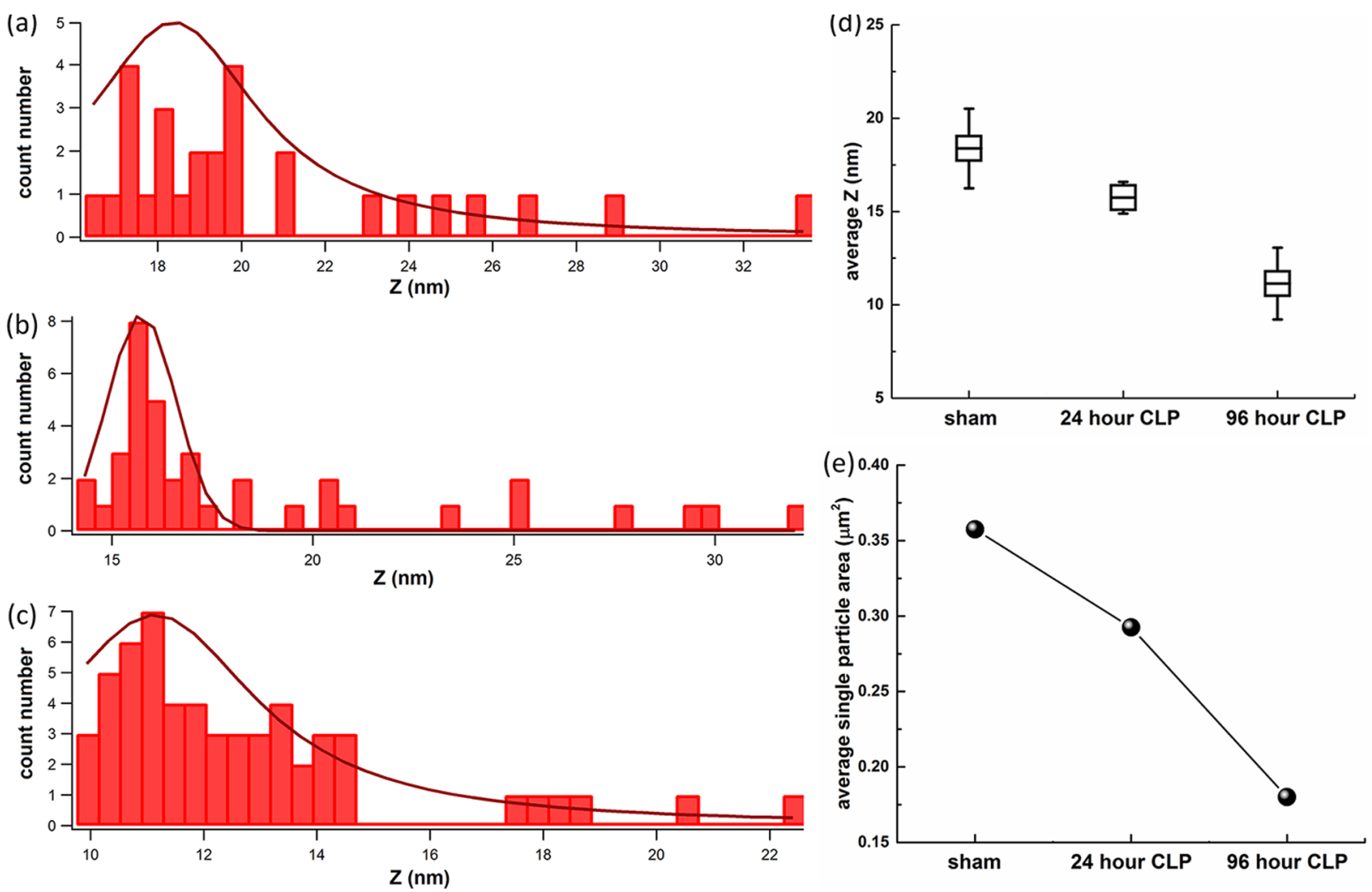

Figure 5. Distribution histograms of the height of the mineral particle from the AM-FM topography images. (a) the sham, (b) $24 \mathrm{~h} \mathrm{CLP,} \mathrm{(c)} 96 \mathrm{~h} C L P$ bone samples, (d) average height with standard deviation, and (e) average single particle area.

The topography image (Figures $3 a-c$ ) show that the size of mineral particles from $96 h C L P$ bone sample is significantly smaller than those from the sham and $24 \mathrm{~h}$ CLP bones. The measured height differences are 151.0, 156.2, and $64.0 \mathrm{~nm}$ for the sham, $24 h C L P$, and $96 h$ CLP bone samples with Ra of $15.95,20.61$, and $9.72 \mathrm{~nm}$, respectively (Table 1 ). Figure $3 \mathrm{~d}-\mathrm{f}$ shows that the contact stiffness of the mineral from the sham, 24 $h C L P$, and $96 h C L P$ bone samples are 0.85-1.59, 0.77-1.47, and $709-988 \mathrm{mN} / \mathrm{m}$, respectively (Table 1$)$. The calculated elastic modulus of mineral from $96 h C L P$ bone sample is 41.373.7 GPa, smaller than the elastic modulus of 68.1-144 and 70.2-134 GPa of the sham and 24h CLP bone samples (Table 1 ). The calculated elastic moduli of the mineral from sham and $24 h$ CLP bone samples are close to the value reported in the literature. $^{55}$ The elastic modulus of mineral shows significant decrease $96 \mathrm{~h}$ after the sepsis insult. The uncertainty analyses (Table S2, SI) reveal that the uncertainty values for elastic modulus are 25.13, 36.02, and $20.18 \mathrm{GPa}$ and uncertainty propagation values are $50.26,72.04$, and $40.35 \mathrm{GPa}$ for the mineral from the sham, $24 h C L P$, and $96 h C L P$ bone samples respectively. For statistical purposes, we compared the elastic modulus of mineral from $10 \mathrm{AM}-\mathrm{FM}$ images obtained from 10 different femurs from the sham, $24 h C L P$, and $96 h C L P$ bones, respectively, as shown in Figure S6 (SI). The mineral from $96 \mathrm{~h}$ CLP bone (Figure S6c, SI) has lower elastic modulus than those from the sham (Figure S6a, SI) and $24 h$ CLP bone samples (Figure S6b, SI). Furthermore, the elastic moduli of the mineral from $24 h$ CLP bone (Figure S6b, SI) and the sham bone (Figure S6a, SI) show negligible difference.

3.4. Histogram Analyses of Collagen Fiber and Mineral. The histogram analyses of the elastic modulus of the collagen fibers and mineral from Figure $1 g-i$ and Figure $3 g-i$ are plotted in Figure $4 \mathrm{a}, \mathrm{c}$, respectively. The cumulative distribution function (CDF) chart for the modulus of the collagen and mineral are plotted in Figure $4 \mathrm{~b}, \mathrm{~d}$, respectively. In Figure $4 \mathrm{a}$, the elastic modulus of collagen fibers from the $24 \mathrm{~h}$ CLP bone is centered at $1.5 \mathrm{GPa}$, much smaller than $6.4 \mathrm{GPa}$ of the sham bone sample. There are two peaks in the histogram of the modulus from the $96 \mathrm{hCLP}$ bone samples, one is centered at $1.8 \mathrm{GPa}$ (corresponding to uncovered part of the collagen fiber) and the other is centered at $6 \mathrm{GPa}$ (corresponding to recovered part of the collagen fiber). These two peaks demonstrate that the mechanical property of collagen fiber has only partially recovered, but not completely recovered to its normal strength $96 \mathrm{~h}$ after the sepsis insult. In Figure 4b, the entire CDF curve of the collagen fibers from $24 h C L P$ bone samples is located at the left side of the sham bone samples, indicating the overall decrease of the elastic modulus of the collagen fibers from the 24 $h C L P$ bones $(P<0.01)$. The CDF curve of collagen fibers from $96 h C L P$ bone samples is located between the CDF curves of the sham and $24 h C L P$ bone samples, revealing the recovery behavior of collagen fiber $(P<0.01)$. For minerals, Figure $4 c, d$ shows that there is slight decrease of elastic modulus from the 24 $h$ CLP bone samples, whereas a distinct decrease can be observed from the $96 h$ CLP bone samples $(P<0.01)$.

3.5. Particle Analysis of Mineral. The distribution histograms with Gaussian fitting from the particle analysis on AM-FM topography images (Figure $3 \mathrm{a}-\mathrm{c}$ ) by using the AFM analysis software (Igor Pro 6.37) are shown in Figure 5a-c, respectively. Figure 5d shows that the average heights of the mineral particles are $18.38 \pm 2.13,15.74 \pm 0.85$, and $11.14 \pm$ $1.92 \mathrm{~nm}$ for the sham, $24 \mathrm{~h} C L P$, and $96 \mathrm{~h}$ CLP bone samples, respectively (Table 1$)$. It reveals that the particle height of the mineral (in $z$-direction) decreases gradually from the sham to 96 


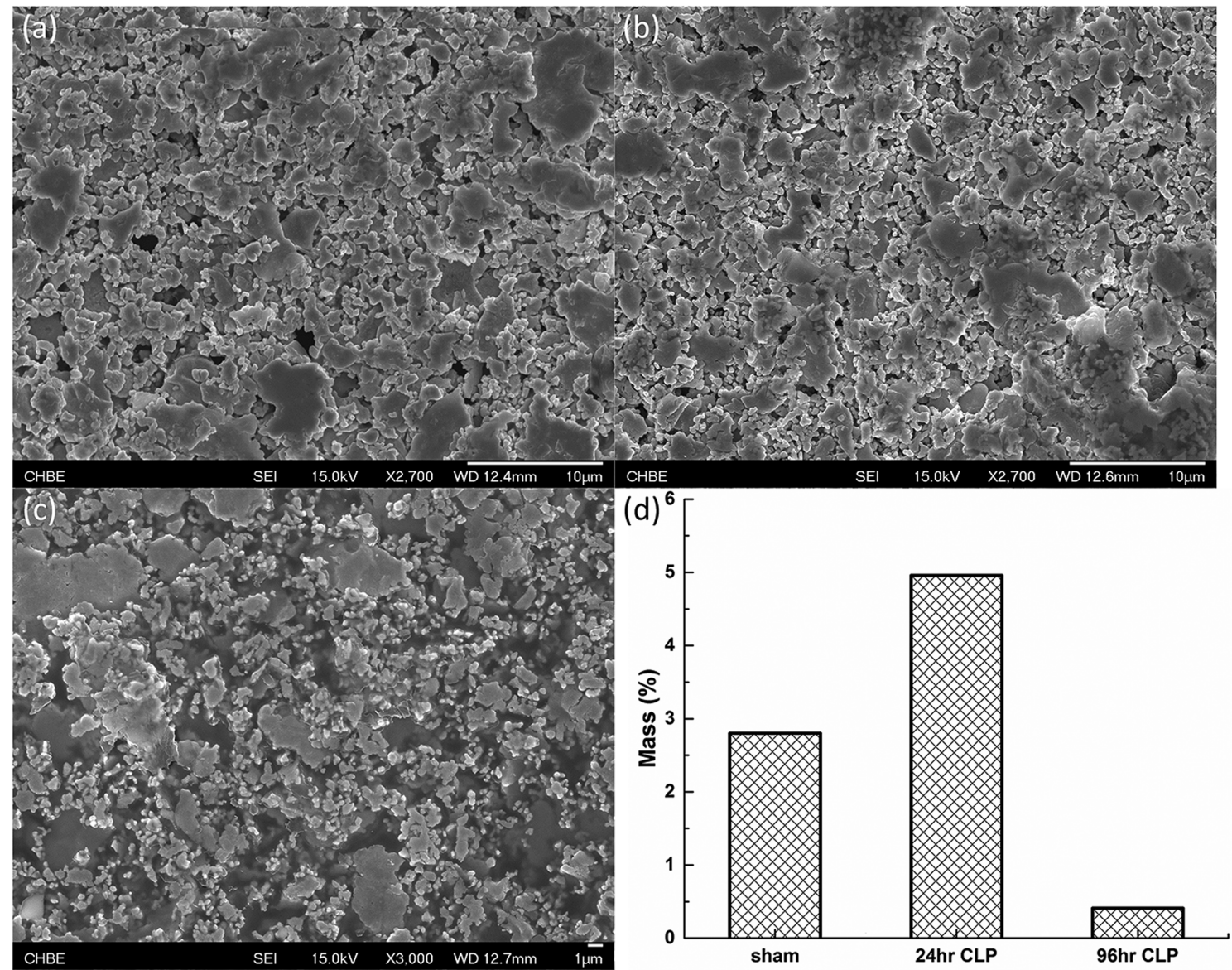

Figure 6. FESEM images of mineral matrix in (a) the sham, (b) $24 \mathrm{~h} \mathrm{CLP,} \mathrm{(c)} 96 \mathrm{~h} C L P$ bone samples, and (d) EDS results of calcium mass in the sham, $24 h C L P$, and $96 h C L P$ bone samples, respectively.

$h$ CLP bone samples. The particle area and the numbers of inplane particles $(x-y$ plane $)$ are also analyzed and summarized in the Figure S7 (SI). The numbers of mineral particles in Figures $3 \mathrm{a}-\mathrm{c}$ are 41,52 and 86 , respectively, and the areas occupied by particles are $58.7 \%, 60.6 \%$, and $61.2 \%$ of the whole image area $(5$ $\mu \mathrm{m} \times 5 \mu \mathrm{m})$ accordingly for the sham, $24 h C L P$, and $96 h C L P$ bone samples, respectively (Table 1 ). Furthermore, Figure 5e shows that the average areas taken up by a single particle are $0.36,0.29$, and $0.18 \mu \mathrm{m}^{2}$ for the sham, $24 h C L P$, and $96 h C L P$ bone samples (Table 1). The average area of the mineral particles from the $96 h C L P$ bone has decreased to about the half of that from the sham bone.

3.6. Field Emission Scanning Electron Microscope Analysis of Mineral. Surface morphology of the mineral and the content of calcium in the mineral are also analyzed by using the field emission scanning electron microscope (FESEM, FEI Quanta 600, Japan) equipped with an energy-dispersive X-ray spectrometer (EDS, Oxford Instruments, U.K.) on the sham and two CLP cortical bones. The FESEM surface morphology images and EDS results are shown in Figure 6. It is difficult to find any difference in term of appearance of the HA crystals $\left(\mathrm{Ca}_{5}\left(\mathrm{PO}_{4}\right)_{5}(\mathrm{OH})\right)$ in the mineral phase between the sham (Figure 6a) and $24 h$ CLP bone (Figure 6b) samples, whereas smaller size of HA crystals and looseness of the mineral structure are observed in the mineral matrix from $96 h$ CLP bone samples (Figure 6c). Moreover, the mass percentage of the calcium in 96 $h$ CLP bone is very low $(0.41 \%)$ according to Figure $6 \mathrm{~d}$. Therefore, mineral density loss happens in the $96 h$ CLP bone samples. Mass percentage of the calcium in $24 h$ CLP bone is $4.96 \%$, higher than those in the sham and $96 h$ CLP bones ( $2.80 \%$ and $0.41 \%$, respectively) (Figure $6 \mathrm{~d}$ ); this suggests that the collagen fibers demineralize $24 \mathrm{~h}$ after the sepsis insult. In fact, bone mineral may act as a reservoir from which the calcium ions can be continually withdrawn for the usage or deposition for storage, as dictated by homeostasis. ${ }^{56}$ In addition, demineralization may influence D-spacing structure quite slightly as the tissues such as tendon and skin showed similar D-spacing compared with the collagen fibers in the normal bones. ${ }^{57,58}$ The response processes of the collagen fibers can be explained by the demineralization of collagen fiber occurring $24 \mathrm{~h}$ after the sepsis insult, leading to an increase of calcium ions in the mineral matrix and the decrease of the elastic modulus of the collagen fiber. However, $96 \mathrm{~h}$ after the sepsis insult, the collagen fiber almost retains its physiological state. Free calcium ions are deposited in hydroxyapatite and attached to stiffen the collagen fibers. The original EDS results including chemical maps and quantitative analyses of mineral matrix of femur cortical bone samples from the sham, $24 h C L P$, and $96 h C L P$ bone samples are shown in Figures S8-S10 (SI).

\section{CONCLUSIONS}

AM-FM technique has been proven capable of offering a fast, direct, and nondestructive way to characterize structure and mechanical property of bone at micro- and nanoscales with ultrahigh resolution (nanometer) and sensitivity. Because bone loss is a complex and gradual process which changes over time, 
our study used two CLP groups ( $24 h$ and $96 h$ ) to measure the changes of the structural, chemical, and mechanical property involving in both mineral and collagen fibers from various bone samples. The findings suggest that both collagen fibers and mineral deteriorate after the sepsis insult, resulting in significant decreases of the elastic modulus approximately $24 \mathrm{~h}$ (for collagen fibers) and $96 \mathrm{~h}$ (for mineral particles) after the sepsis insult, respectively. It should be noticed that the elastic modulus reduction in mineral may be associated with decreasing of whole bone strength. These results suggest that the mechanical change of the collagen fiber starts much earlier and is not reflected by changes of macroscopic bone strength. Hence the whole bone strength reduction or bone density loss is not enough to monitor the bone diseases. The mechanical property of collagen fibers may be a better indicator of bone diseases in the early stage. In addition, the sepsis insult causes the nanoscale structural and chemical changes of both collagen fiber and mineral. Our study focuses on characterizing the nanoscale structure and mechanical property of bone microconstituents, which offers an indicator for early diagnosis of bone disease. The findings may provide new mechanistic information about sepsis-related bone diseases.

\section{ASSOCIATED CONTENT}

\section{S Supporting Information}

Electronic Supporting Information (ESI) available: including following: . The Supporting Information is available free of charge on the ACS Publications website at DOI: 10.1021/ acsbiomaterials.8b01087.

Principles of AM-FM technique; calculations of uncertainty and uncertainty propagation; calculations of Hamaker constant $(H)$ and minimum distance of approach per cycle $\left(d_{\min }\right)$ of collagen fibers; Figures S1-S10; Tables S1-S3; references (PDF)

\section{AUTHOR INFORMATION}

\section{Corresponding Author}

*E-mail: mpezk@nus.edu.sg.

ORCID $\odot$

Yao Sun: 0000-0001-8802-0580

Kaiyang Zeng: 0000-0002-3348-0018

Notes

The authors declare no competing financial interest.

\section{ACKNOWLEDGMENTS}

The authors thank the financial support by National University of Singapore through the Eng-Med Seed Grant (R-265-000-532112) and Ministry of Education, Singapore, through National University of Singapore on Academic Research Funding (AcRF) R-265-000-495-112. Y.S. thanks the support of research scholarship from National University of Singapore.

\section{REFERENCES}

(1) Zhao, F.; Yin, Y.; Lu, W. W.; Leong, J. C.; Zhang, W.; Zhang, J.; Zhang, M.; Yao, K. Preparation and Histological Evaluation of Biomimetic Three-Dimensional Hydroxyapatite/Chitosan-Gelatin Network Composite Scaffolds. Biomaterials 2002, 23 (15), 3227-3234.

(2) Chen, P.-Y.; Novitskaya, E.; Lopez, M. I.; Sun, C.-Y.; McKittrick, J. Toward a Better Understanding of Mineral Microstructure in Bony Tissues. Bioinspired, Biomimetic Nanobiomater. 2014, 3 (2), 71-84.
(3) Burstein, A.; Zika, J.; Heiple, K.; Klein, L. Contribution of Collagen and Mineral to the Elastic-Plastic Properties of Bone. J. Bone Jt. Surg. 1975, 57 (7), 956-961.

(4) Rho, J.-Y.; Kuhn-Spearing, L.; Zioupos, P. Mechanical Properties and the Hierarchical Structure of Bone. Med. Eng. Phys. 1998, 20 (2), 92-102.

(5) Seeman, E. Bone Quality: the Material and Structural Basis of Bone Strength. J. Bone Miner. Metab. 2008, 26 (1), 1-8.

(6) Hamed, E.; Novitskaya, E.; Li, J.; Chen, P. Y.; Jasiuk, I.; McKittrick, J. Elastic Moduli of Untreated, Demineralized and Deproteinized Cortical Bone: Validation of a Theoretical Model of Bone as an Interpenetrating Composite Material. Acta Biomater. 2012, 8 (3), 1080-1092.

(7) Wallace, J. M.; Orr, B. G.; Marini, J. C.; Banaszak Holl, M. M. Nanoscale Morphology of Type I Collagen is Altered in the Brtl Mouse Model of Osteogenesis Imperfecta. J. Struct. Biol. 2011, 173 (1), 146152.

(8) Dubey, D. K.; Tomar, V. Role of Molecular Level Interfacial Forces in Hard Biomaterial Mechanics: A Review. Ann. Biomed. Eng. 2010, 38 (6), 2040-2055.

(9) Terashima, A.; Okamoto, K.; Nakashima, T.; Akira, S.; Ikuta, K.; Takayanagi, H. Sepsis-Induced Osteoblast Ablation Causes Immunodeficiency. Immunity 2016, 44 (6), 1434-1443.

(10) Abe, T.; Shin, J.; Hosur, K.; Udey, M. C.; Chavakis, T.; Hajishengallis, G. Regulation of Osteoclast Homeostasis and Inflammatory Bone Loss by MFG-E8. J. Immunol. 2014, 193 (3), 1383-1391.

(11) Puthucheary, Z. A.; Sun, Y.; Zeng, K.; Vu, L. H.; Zhang, Z. W.; Lim, R. Z. L.; Chew, N. S. Y.; Cove, M. E. Sepsis Reduces Bone Strength Before Morphologic Changes Are Identifiable. Crit. Care Med. 2017, 45 (12), e1254-e1261.

(12) Orford, N. R.; Lane, S. E.; Bailey, M.; Pasco, J. A.; Cattigan, C.; Elderkin, T.; Brennan-Olsen, S. L.; Bellomo, R.; Cooper, D. J.; Kotowicz, M. A. Changes in Bone Mineral Density in the Year after Critical Illness. Am. J. Respir. Crit. Care Med. 2016, 193 (7), 736-744.

(13) Lin, P.-C.; Lin, S.; Wang, P. C.; Sridhar, R. Techniques for Physicochemical Characterization of Nanomaterials. Biotechnol. Adv. 2014, 32 (4), 711-726.

(14) Mandair, G. S.; Morris, M. D. Contributions of Raman Spectroscopy to the Understanding of Bone Strength. BoneKEy Rep. 2015, 4, 620

(15) Kalender, W.; Engelke, K.; Fuerst, T. P.; Glüer, C.-C.; Laugier, P.; Shepherd, J. 7. Quantitative Ultrasound. J. ICRU 2009, 9 (1), 71-104.

(16) Zysset, P. K.; Edward Guo, X.; Edward Hoffler, C.; Moore, K. E.; Goldstein, S. A. Elastic Modulus and Hardness of Cortical and Trabecular Bone Lamellae Measured by Nanoindentation in the Human Femur. J. Biomech. 1999, 32 (10), 1005-1012.

(17) Hoffler, C. E.; Guo, X. E.; Zysset, P. K.; Goldstein, S. A. An Application of Nanoindentation Technique to Measure Bone Tissue Lamellae Properties. J. Biomech. Eng. 2005, 127 (7), 1046-1053.

(18) Rho, J.-Y.; Tsui, T. Y.; Pharr, G. M. Elastic Properties of Human Cortical and Trabecular Lamellar Bone Measured by Nanoindentation. Biomaterials 1997, 18 (20), 1325-1330.

(19) Zbib, A. A.; Bahr, D. F. Dislocation Nucleation and Source Activation during Nanoindentation Yield Points. Metall. Mater. Trans. A 2007, 38 (13), 2249-2255.

(20) Variola, F. Atomic Force Microscopy in Biomaterials Surface Science. Phys. Chem. Chem. Phys. 2015, 17 (5), 2950-2959.

(21) Balooch, G.; Balooch, M.; Nalla, R. K.; Schilling, S.; Filvaroff, E. H.; Marshall, G. W.; Marshall, S. J.; Ritchie, R. O.; Derynck, R.; Alliston, T. TGF- $\beta$ Regulates the Mechanical Properties and Composition of Bone Matrix. Proc. Natl. Acad. Sci. U. S. A. 2005, 102 (52), 1881318818.

(22) Tao, N. J.; Lindsay, S. M.; Lees, S. Measuring the Microelastic Properties of Biological Material. Biophys. J. 1992, 63 (4), 1165-1169.

(23) Stolz, M.; Raiteri, R.; Daniels, A. U.; VanLandingham, M. R.; Baschong, W.; Aebi, U. Dynamic Elastic Modulus of Porcine Articular Cartilage Determined at Two Different Levels of Tissue Organization 
by Indentation-Type Atomic Force Microscopy. Biophys. J. 2004, 86 (5), 3269-3283.

(24) Milovanovic, P.; Potocnik, J.; Djonic, D.; Nikolic, S.; Zivkovic, V.; Djuric, M.; Rakocevic, Z. Age-Related Deterioration in Trabecular Bone Mechanical Properties at Material Level: Nanoindentation Study of the Femoral Neck in Women by Using AFM. Exp. Gerontol. 2012, 47 (2), 154-159.

(25) Wenger, M. P. E.; Bozec, L.; Horton, M. A.; Mesquida, P. Mechanical Properties of Collagen Fibrils. Biophys. J. 2007, 93 (4), $1255-1263$.

(26) Campbell, S. E.; Ferguson, V. L.; Hurley, D. C. Nanomechanical Mapping of the Osteochondral Interface with Contact Resonance Force Microscopy and Nanoindentation. Acta Biomater. 2012, 8 (12), 4389-4396.

(27) Garcia, R.; Herruzo, E. T. The Emergence of Multifrequency Force Microscopy. Nat. Nanotechnol. 2012, 7 (4), 217-226.

(28) Martínez, N. F.; Lozano, J. R.; Herruzo, E. T.; Garcia, F.; Richter, C.; Sulzbach, T.; Garcia, R. Bimodal Atomic Force Microscopy Imaging of Isolated Antibodies in Air and Liquids. Nanotechnology 2008, 19 (38), 384011.

(29) Solares, S. D.; Chawla, G. Multi-Frequency Atomic Force Microscopy Combining Amplitude- and Frequency-Modulation Techniques. MRS Online Proceedings Library Archive 2012, 1422, mrsf11-1422-qq03-07.

(30) Dietz, C.; Schulze, M.; Voss, A.; Riesch, C.; Stark, R. W. Bimodal Frequency-Modulated Atomic Force Microscopy with Small Cantilevers. Nanoscale 2015, 7 (5), 1849-1856.

(31) Santos, S.; Lai, C.-Y.; Olukan, T.; Chiesa, M. Multifrequency AFM: From Origins to Convergence. Nanoscale 2017, 9 (16), 50385043.

(32) Ricci, M.; Quinlan, R. A.; Voïtchovsky, K. Sub-Nanometre Mapping of the Aquaporin-Water Interface with Multifrequency Atomic Force Microscopy. Soft Matter 2017, 13 (1), 187-195.

(33) Kocun, M.; Labuda, A.; Meinhold, W.; Revenko, I.; Proksch, R. Fast, High Resolution, and Wide Modulus Range Nanomechanical Mapping with Bimodal Tapping Mode. ACS Nano 2017, 11 (10), 10097-10105.

(34) Harcombe, D. M.; Ruppert, M. G.; Ragazzon, M. R. P.; Fleming, A. J. Lyapunov Estimation for High-Speed Demodulation in Multifrequency Atomic Force Microscopy. Beilstein J. Nanotechnol. 2018, 9, 490-498.

(35) Meier, T.; et al. Multifrequency Force Microscopy Using Flexural and Torsional Modes by Photothermal Excitation in Liquid: Atomic Resolution Imaging of Calcite (1014). Nanotechnology 2016, 27 (8), No. 085702.

(36) Shamsudhin, N.; Rothuizen, H.; Nelson, B. J.; Sebastian, A. Multi-Frequency Atomic Force Microscopy: A System-Theoretic Approach. IFAC Proc. Vol. 2014, 47 (3), 7499-7504.

(37) Al-Rekabi, Z.; Contera, S. Multifrequency AFM Reveals Lipid Membrane Mechanical Properties and the Effect of Cholesterol in Modulating Viscoelasticity. Proc. Natl. Acad. Sci. U. S. A. 2018, 115 (11), 2658-2663.

(38) Chawla, G.; Solares, S. D. Mapping of Conservative and Dissipative Interactions in Bimodal Atomic Force Microscopy Using Open-Loop and Phase-Locked-Loop Control of the Higher Eigenmode. Appl. Phys. Lett. 2011, 99 (7), No. 074103.

(39) Sun, Y.; Hu, Z.; Zhao, D.; Zeng, K. Mechanical Properties of Microcrystalline Metal-Organic Frameworks (MOFs) Measured by Bimodal Amplitude Modulated-Frequency Modulated Atomic Force Microscopy. ACS Appl. Mater. Interfaces 2017, 9 (37), 32202-32210.

(40) Li, T.; Chang, S.-W.; Florez, N. R.; Buehler, M. J.; Shefelbine, S.; Dao, M.; Zeng, K. Studies of Chain Substitution Caused Sub-Fibril Level Differences in Stiffness and Ultrastructure of Wildtype and Oim/ Oim Collagen Fibers Using Multifrequency-AFM and Molecular Modeling. Biomaterials 2016, 107, 15-22.

(41) Santos, S.; Guang, L.; Souier, T.; Gadelrab, K.; Chiesa, M.; Thomson, N. H. A Method to Provide Rapid in situ Determination of Tip Radius in Dynamic Atomic Force Microscopy. Rev. Sci. Instrum. 2012, 83 (4), No. 043707.
(42) Kopycinska-Müller, M.; Geiss, R. H.; Hurley, D. C. Contact Mechanics and Tip Shape in AFM-Based Nanomechanical Measurements. Ultramicroscopy 2006, 106 (6), 466-474.

(43) Adams, G. G.; Nosonovsky, M. Contact Modeling — Forces. Tribol. Int. 2000, 33 (5-6), 431-442.

(44) Ebeling, D.; Solares, S. D. Bimodal Atomic Force Microscopy Driving the Higher Eigenmode in Frequency-Modulation Mode: Implementation, Advantages, Disadvantages and Comparison to the Open-Loop Case. Beilstein J. Nanotechnol. 2013, 4, 198-207.

(45) Rodríguez, T. R.; García, R. Compositional Mapping of Surfaces in Atomic Force Microscopy by Excitation of the Second Normal Mode of the Microcantilever. Appl. Phys. Lett. 2004, 84 (3), 449-451.

(46) Garcia, R.; Proksch, R. Nanomechanical Mapping of Soft Matter by Bimodal Force Microscopy. Eur. Polym. J. 2013, 49 (8), 1897-1906.

(47) Wagner, W.; et al. Uncertainty Quantification in Nanomechanical Measurements Using the Atomic Force Microscope. Nanotechnology 2011, 22 (45), 455703.

(48) Lai, C.-Y.; Perri, S.; Santos, S.; Garcia, R.; Chiesa, M. Rapid Quantitative Chemical Mapping of Surfaces with Sub-2 nm Resolution. Nanoscale 2016, 8 (18), 9688-9694.

(49) Lai, C. Y.; Sergio, S.; et al. Reconstruction of Height of SubNanometer Steps with Bimodal Atomic Force Microscopy. Nanotechnology 2016, 27 (7), No. 075701.

(50) Lai, C.-Y.; Santos, S.; Chiesa, M. Systematic Multidimensional Quantification of Nanoscale Systems From Bimodal Atomic Force Microscopy Data. ACS Nano 2016, 10 (6), 6265-6272.

(51) Chiou, Y.-C.; Olukan, T. A.; Almahri, M. A.; Apostoleris, H.; Chiu, C. H.; Lai, C.-Y.; Lu, J.-Y.; Santos, S.; Almansouri, I.; Chiesa, M. Direct Measurement of the Magnitude of the van der Waals Interaction of Single and Multilayer Graphene. Langmuir 2018, 34 (41), 1233512343 .

(52) Perumal, S.; Antipova, O.; Orgel, J. P. R. O. Collagen Fibril Architecture, Domain Organization, and Triple-Helical Conformation Govern Its Proteolysis. Proc. Natl. Acad. Sci. U. S. A. 2008, 105 (8), 2824-2829.

(53) Orgel, J. P. R. O.; Irving, T. C.; Miller, A.; Wess, T. J. Microfibrillar Structure of Type I Collagen in situ. Proc. Natl. Acad. Sci. U. S. A. 2006, 103 (24), 9001-9005.

(54) van der Rijt, J. A. J.; van der Werf, K. O.; Bennink, M. L.; Dijkstra, P. J.; Feijen, J. Micromechanical Testing of Individual Collagen Fibrils. Macromol. Biosci. 2006, 6 (9), 697-702.

(55) Saber-Samandari, S.; Gross, K. A. Micromechanical Properties of Single Crystal Hydroxyapatite by Nanoindentation. Acta Biomater. 2009, 5 (6), 2206-2212.

(56) Carmona, R. H. Bone Health and Osteoporosis; Office of the Surgeon General: Washington, DC, 2004.

(57) Fang, M.; Liroff, K. G.; Turner, A. S.; Les, C. M.; Orr, B. G.; Holl, M. M. B. Estrogen Depletion Results in Nanoscale Morphology Changes in Dermal Collagen. J. Invest. Dermatol. 2012, 132 (7), 17911797.

(58) Wallace, J. M.; Chen, Q.; Fang, M.; Erickson, B.; Orr, B. G.; Banaszak Holl, M. M. Type I Collagen Exists as a Distribution of Nanoscale Morphologies in Teeth, Bones, and Tendons. Langmuir 2010, 26 (10), 7349-7354. 